\title{
Detection and study of the compact HII region N26A-B in the Small Magellanic Cloud
}

\begin{abstract}
G. Testor ${ }^{\star}$
Observatoire de Paris, section de Meudon, DAEC, 92195 Meudon Cedex, France

Received 12 January 2001 / Accepted 15 March 2001

Abstract. This paper presents new imagery and spectrophotometric results for the N26 HII region in the Small Magellanic Cloud. The observations using monochromatic images and low-resolution spectra (3700-10000 $\AA$ ) reveal a compact and complex nebula composed of two cores $\mathrm{A}$ and $\mathrm{B}$ where $\mathrm{A}$ in the region of $\mathrm{H} \beta$ is brighter than B by a factor $\sim 5$ and distance of $2^{\prime \prime}$. The core A of $F W H M \sim 2$.' 1 or $0.6 \mathrm{pc}$ presents a high excitation [O III] $\lambda \lambda 5007+4959 / \mathrm{H} \beta$ up to $\sim 8$ and a high reddening $E(B-V) \leq 0.6$, while the core $\mathrm{B}$ is less excited but has a higher reddening $\geq 0.8$. Each core contains one exciting source; the brighter one should be responsible for the high excitation of $\mathrm{A}$. The apparent spectral type of the two cores ranges from $\mathrm{O} 7$ to $\mathrm{O} 9 \mathrm{~V}$ and the gas electron density and temperature were derived from the absorption and emission-line intensities. The total mass of the ionized gas is evaluated at $13 M_{\odot}$. The chemical abundances of $\mathrm{He}, \mathrm{O}, \mathrm{N}, \mathrm{Ne}, \mathrm{S}$, and Ar were computed. These abundances seem consistent with average abundances for SMC HII regions, except N that appears slightly overabundant. N26A-B is comparable to the objects previously observed in the LMC and SMC that we have called "blobs".
\end{abstract}

Key words. galaxies: Magellanic Clouds - ISM: individual objects: N26A-B, N25 (SMC) - ISM: HII regions ISM: dust, extinction - stars: early-type

\section{Introduction}

High-excitation compact HII regions in the Small Magellanic Cloud (SMC) and the Large Magellanic Cloud (LMC) that we have named "blobs" (Heydari-Malayeri \& Testor 1982) are among the brightest objects in the Clouds and belong to more extended emission nebulae detected by Henize (1956).

Their compact and complex morphology was pointed out only two decades ago by electronographic and CCD imagery and then by IDS and CCD spectroscopy in the framework of a study of individual young HII regions in an environment of low metallicity.

Up to now, five blobs are known in the LMC: N159A, N11A, N160A1 \& A2 and N83-B, (Heydari-Malayeri \& Testor 1982, 1983, 1985, 1986; Heydari-Malayeri et al. 1990) and two in the SMC: N88A (Testor \& Pakull 1985) and N81 (Heydari-Malayeri et al. 1988).

As the clouds are close enough for us to observe individual stars and distant enough to simultaneously collect a large proportion of a small extended object in the spectrograph slit, the blobs are the best candidates to study individual star forming regions both globally and in detail. According to the HII region classification (Wilcots 1994)

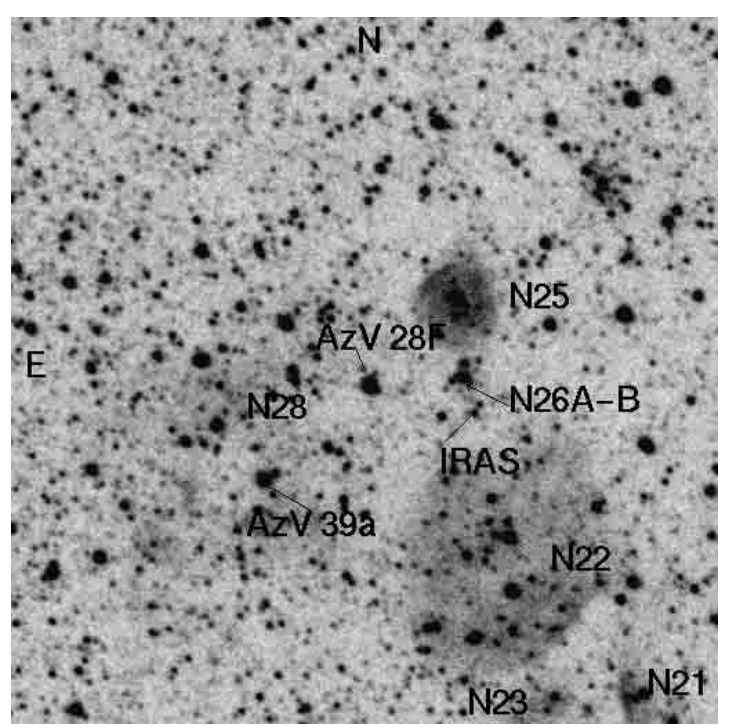

Fig. 1. ESO/U field of $6^{\prime} \times 6^{\prime}$. Extended HII regions around the HII region N26 are labelled. N26 containing the compact HII region, the stars $\mathrm{AzV} 28 \mathrm{~F}$, the Wolf-Rayet star $\mathrm{AzV}$ 39a (WN4.5+O4, $m=14.43$ ) and the IRAS source: IRAS 004627331 are indicated by an arrow.

\footnotetext{
* e-mail: gerard.testor@obspm.fr
} 


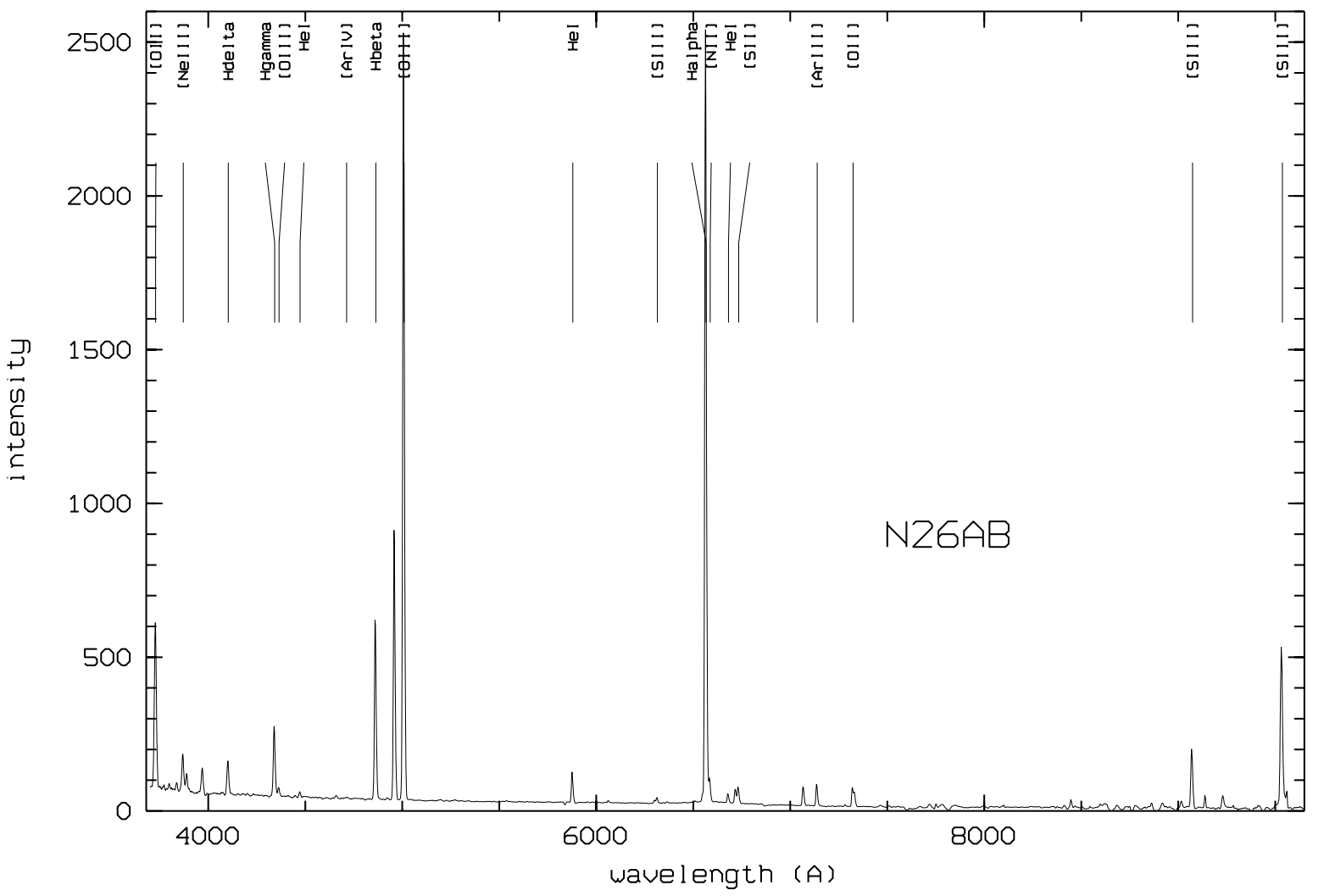

Fig. 2. The plot shows E-W matched red and blue CCD spectra obtained in 1990 and 1997. For display and spectral resolution uniformity purposes the optical data have been degraded by Gaussian-filtering. The intensity is units of $10^{-16} \mathrm{ergs}^{-2} \mathrm{~s}^{-1} \AA^{-1}$.

these suitable HII reference regions ionized mainly by a massive star should enter a category between the dense (type III) and classical (type IV) HII regions.

This paper, using imagery and long-slit spectroscopy, reports the detection of a new blob in the emission nebula N26 (Henize 1956) in the Small Magellanic Cloud (SMC). On the ESO/U image (Fig. 1) the nebula N26 is a bright region located in a zone of absorption and appears as a fuzzy stellar image. N26 is surrounded by several Henize extended HII regions: N21, N22, N23, N25 and N28. In Davies et al. (1976), N26 and N25 belong to the same HII region of $1.2^{\prime} \times 1^{\prime}$, called DEM38, where a high Balmer decrement $\mathrm{H} \alpha / \mathrm{H} \beta=3.78$ was found by Caplan \& Deharveng (1986) through an aperture of $\phi 1^{\prime}$.

The north nebula N25 $\left(\phi \sim 20^{\prime \prime}\right)$ studied by Hutching \& Thomson (1988) is ionized by a bright star of type O9 and N28 contains the Wolf-Rayet AzV 39a of type WN4.5 (Azzopardi \& Vigneau 1979). N26 is located at the western edge of a strong HI concentration (McGee et al. 1981) and in the high resolution IRAS images at $12 \mu$ and $25 \mu$ it coincides with an intense IR emission corresponding to a region of maximum dust-to-gas ratio (Stanimirovic et al. 2000). N26 lies $\sim 15^{\prime \prime}$ north-west of the source IRAS 00462 7331 and $8^{\prime}$ south-west of the brightest CO sources associated with the HII region N12 and N27 in the SMC (Israel et al. 1993). N26 was catalogued as a planetary nebula by Webster (1976), and Pagel et al. (1978) found an oxygen abundance of $12+\log (\mathrm{O} / \mathrm{H})=8.09$. This paper is arranged as follows:

- in Sect. 2, we give a brief description of the data base concerning the compact HII region N26A-B, and of the nebula N25, accumulated by the author during the last decade, consisting of $U B V$ and monochromatic imagery and optical and near infra-red (NIR) spectrophotometry and data processing;

- in Sect. 3 the morphology and physical characteristics especially of the compact region are described: extinction, excitation, density, temperature and mass of ionized gas;

- in Sect. 4 we describe the chemical abundances, followed by a summary of results.

Table 1. Journal of the spectrographic observations with the B\&C.

\begin{tabular}{|c|c|c|c|c|c|c|}
\hline Id & Date & $\begin{array}{r}\text { Expo. } \\
(\mathrm{sec})\end{array}$ & $\begin{array}{l}\text { Grating } \\
(\AA / \mathrm{mm})\end{array}$ & $\begin{array}{l}\text { Range } \\
(\AA)\end{array}$ & $\begin{array}{l}\text { Slit Width } \\
\left({ }^{\prime \prime}\right)\end{array}$ & $\begin{array}{l}F W H M \\
(\AA)\end{array}$ \\
\hline N26 & 06/01/90 & 3600 & 228 & 6500-9900 & 3 & 9.5 \\
\hline $\mathrm{N} 25$ & $29 / 10 / 91$ & 1800 & 114 & $3700-7400$ & 2 & 4.8 \\
\hline N26 & $29 / 10 / 91$ & 1800 & & & & \\
\hline N26 & $29 / 10 / 91$ & 1800 & & & & \\
\hline $\mathrm{N} 26$ & 09/11/91 & 1800 & & & & \\
\hline N26 & $14 / 12 / 97$ & 600 & 114 & $3700-7400$ & 4 & 7.3 \\
\hline
\end{tabular}




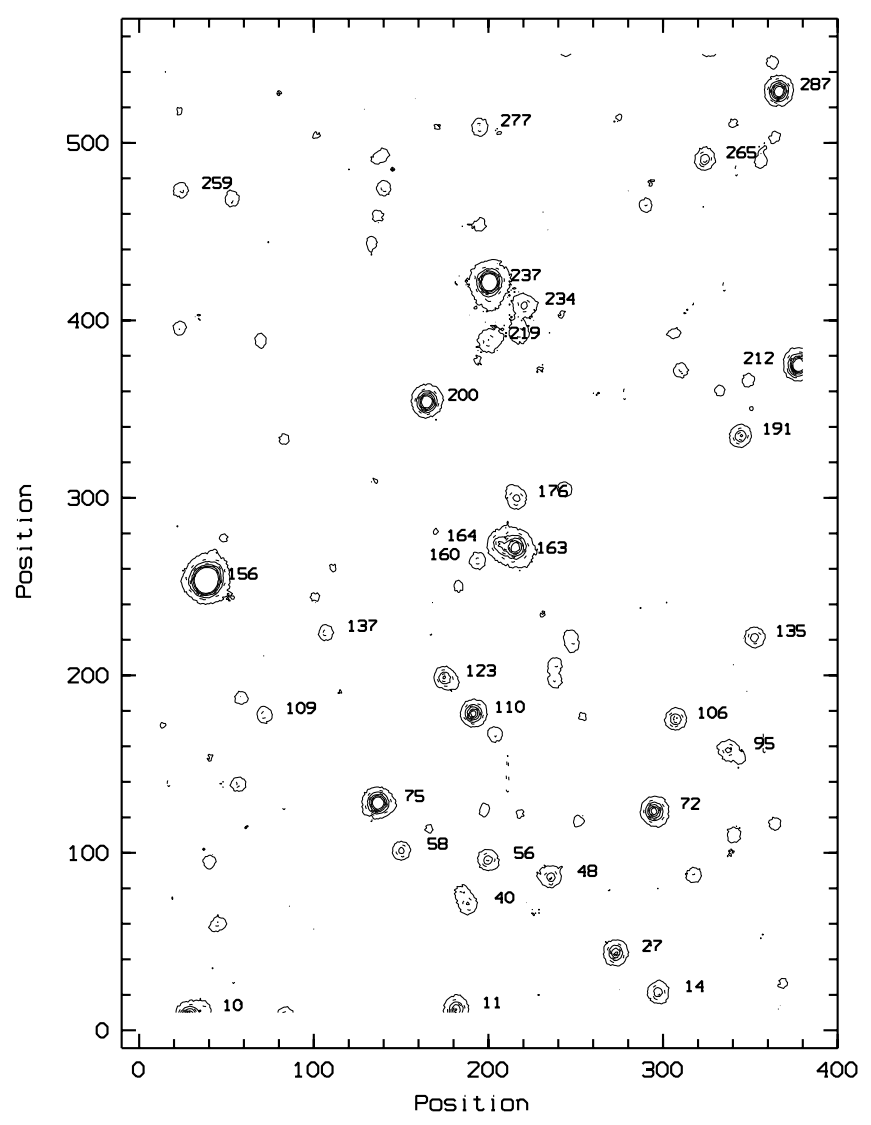

Fig. 3. Field of N26A-B $\left(107^{\prime \prime} \times 152^{\prime \prime}\right)$ obtained through the $V$ filter. The stars of magnitude $<17.5$ are labelled. The stars \#163 and \#164 are separated by $2^{\prime \prime}$. North is up and East is left.

\section{Observations and reductions}

\subsection{Spectroscopy}

Table 1 lists all the observations of long-slit optical and NIR spectra obtained during three observing runs at the ESO $1.5 \mathrm{~m}$ telescope with the B\&C spectrograph using:

- in January 1990, the CCD \#9 (GEC), $1000 \times$ 1000 pixels, each pixel being $22 \mu \times 22 \mu$, corresponding to $1^{\prime \prime} \times 1^{\prime \prime}$ on the sky, and using the grating \#18 of dispersion $228 \AA \mathrm{mm}^{-1}$ allowing observations of the nebular transitions [S III] at $9069 \AA$ and $9532 \AA$ with an effective spectral resolution $(F W H M)$ of $9.5 \AA$;

- in October 1991, a high resolution $2000 \times 2000$ Ford $\mathrm{CCD}$ at $15 \mu \times 15 \mu$ per pixel corresponding to $0^{\prime \prime} .7 \times 0.0^{\prime \prime} 7$ on the sky and the grating \#23 with a dispersion of $114 \AA \mathrm{mm}^{-1}$ was used, giving a large spectral coverage from 3600 to $7400 \AA$ and a $F W H M$ of $4.8 \AA$;

- in December 1997, the CCD \#39 (Loral) was used, with $2048 \times 2048$ pixels binned by a factor 2 in the spatial direction providing an image scale of $1^{\prime \prime} 55 /$ pixel and the grating \#23 with a dispersion of $126 \AA \mathrm{mm}^{-1}$ from 3600 to $7400 \AA$ giving a $F W H M$ of $\sim 7.3 \AA$. The frames were obtained through $2^{\prime \prime}, 3^{\prime \prime}$ and $4^{\prime \prime}$ long-slit widths oriented E-W.
All the spectroscopic raw data were reduced mainly with the Midas software package LONG available at the Paris-Meudon Observatory. The usual corrections, such as flatfielding and sky substraction, were applied in a standard way. The spectra were wavelength calibrated with a helium-argon arc. The flux was calibrated using observations of the spectrophotometric standard stars Feige 110 and LTT2415 (Oke 1974).

From the CCD frames, one-dimensional spectra were extracted by summing a range of pixels along the long-slit corresponding to the full width of the nebulae $\left(\sim 7^{\prime \prime}\right.$ for the compact HII region). The 1-d spectrum corresponding to the period 91 was obtained from averaged frames.

The line strengths corrected for the extinction were measured by integrating the fluxes under the emissionline profiles. The uncertainty estimates including photon counting, detector noise and noise due to sky substraction give for the [O III] $\lambda 4363$ emission line a $S / N$ in the range of $20-25$. This $S / N$ was calculated using the method given by Newberry (1994). The uncertainties are combined with those due to the standard star and reddening correction not easily evaluable, depending on the position in the spectral range and estimated to be approximatively 1-3\%. Using an approximate spectral type of O7 based on our spectral type determination, the Balmer lines in N26A-B were corrected for an assumed stellar absorption of an equivalent width $(E W)$ of $\sim 2 \AA$ in each line (Skillman 1985), corresponding to $\sim 0.2 \%$ of the emission line strength in $\mathrm{H} \alpha$. The reddening-uncorrected lines normalized to the $\mathrm{H} \beta$ flux are listed in Table 5 with their $S / N$. In the abundance computations, the [Ar IV] $\lambda 4711$ intensity strongly blended with He I $\lambda 4713$ was derived using the theoretical ratios for hydrogen and helium from Hummer \& Storey (1987). Figure 2 displays the extracted one-dimensional NIR and optical long-slit spectra of N26 A-B merged at $\lambda 7400$ obtained in 1990 and 1997 respectively.

\section{2. $C C D$ imagery}

Narrow-band optical images of the N26 field and $U B V$ images of the standard star field E7 (Graham 1982) were taken in October 1987 using the $1000 \times 1000$ CCD GEC

Table 2. Log of CCD images.

\begin{tabular}{llllllll}
\hline \hline Object & Filter & $\begin{array}{l}\lambda \\
(\AA)\end{array}$ & $\begin{array}{l}\delta \lambda \\
(\AA)\end{array}$ & $\begin{array}{l}\text { Expo. } \\
(\mathrm{s})\end{array}$ & Tel. & Date & $\begin{array}{l}\text { Seeing } \\
\text { (arcsec) }\end{array}$ \\
\hline N26 & $U$ & 3560 & 510 & 900 & $2.2 \mathrm{~m}$ & $2 / 9 / 1987$ & 1.5 \\
& $B$ & 4350 & 1000 & 180 & & & \\
& $V$ & 5550 & 1000 & 90 & & & \\
& {$[\mathrm{O}$ II $]$} & 3727 & 50 & 1800 & & & \\
& {$[\mathrm{O}$ III $]$} & 5007 & 10 & 900 & & & \\
& $\mathrm{H} \beta$ & 4861 & 10 & 900 & & & \\
& He II & 4686 & 20 & 1200 & & & \\
E7M & $U$ & 3560 & 510 & $2 \times 240$ & & \\
& $B$ & 4350 & 1000 & $2 \times 60$ & & & \\
& $V$ & 5550 & 1000 & $2 \times 30$ & & & \\
\hline
\end{tabular}


Table 3. Photometry of the stars towards N26 under the magnitude $V=17.5$.

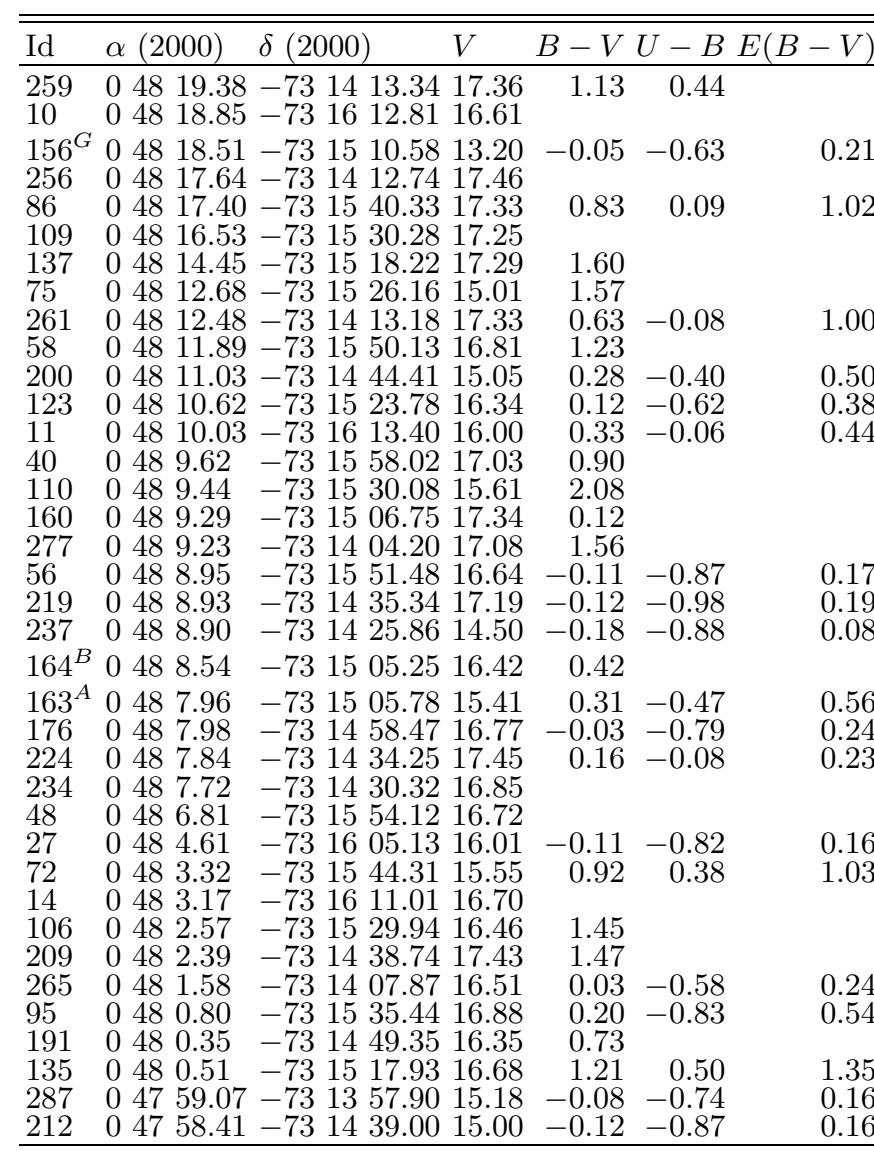

${ }^{G}$ Star AzV 28F (B5 Iab), our spectrum confirms its galactic classification

${ }^{A}$ and ${ }^{B}$ In the DENIS survey \#163 and \#164 are labelled DMC J004807.94-731505.7 and DMC J004808.29-731505.0 respectively. This survey gives $I=14.45$ and $K=11.96$ for the two stars, which might not be spatially resolved. The IRAS source 00462-7331 lies $\sim 15^{\prime \prime}$ south-east of N26A-B.

at a pixel scale of 0 '. 26 per pixel attached to the $2.2 \mathrm{~m}$ telescope at la Silla. The CCD images were reduced using MIDAS image processing system. Table 2 summarizes the $\log$ of CCD frames.

\subsection{Photometry}

The single channel photometer was used at the Cassegrain focus of the ESO $1 \mathrm{~m}$ telescope during the same period in October 87, allowing us to obtain the $U B V$ of two stars in the field of N26 that will be used as photometric standards. Their $V$ magnitudes and colors were measured with the ESO Snoopy program and are labelled 156 and 200 in Fig. 3. The reduction of the other stars contained in the N26 field was done from the $U B V$ frames using DAOPHOT/ALLSTAR (Stetson 1987) that fits the profiles of the stars. A color correction was applied using the stars $\mathrm{m}, \mathrm{n}$ and $\mathrm{s}$ observed in the standard $\mathrm{E} 7$ region and the final calibration was obtained using our standard stars \#156 and \#200. The photometric properties of the stars
Table 4. Coordinates, mean reddening, mean excitation ([O III $] \lambda \lambda 5007+4959 / \mathrm{H} \beta$ ) and size of the whole nebula along the slit in $\mathrm{H} \beta$. The $V$ magnitude of the ionizing source is derived from imagery.

\begin{tabular}{|c|c|c|c|c|c|c|c|}
\hline Regions & $\alpha(2000)$ & $\delta(2000)$ & $\frac{\mathrm{H} \alpha}{\mathrm{H} \beta}$ & $\frac{[O I I I]}{\mathrm{H} \beta}$ & \multicolumn{2}{|c|}{$F W H M$} & $V$ \\
\hline N26 & 0 & 1505.8 & 4.80 & 6. & 2.6 & 0.8 & 15.0 \\
\hline $\begin{array}{l}\text { N26A-B } \\
\text { N25 }\end{array}$ & 0488.9 & 31425.9 & $\begin{array}{l}4.29 \\
2.86\end{array}$ & $\begin{array}{l}5.7 \\
4.2\end{array}$ & $\begin{array}{l}4.0 \\
27\end{array}$ & $\begin{array}{l}1.2 \\
8.1\end{array}$ & 14.50 \\
\hline
\end{tabular}

${ }^{n}$ slit width $=2^{\prime \prime},{ }^{l}$ slit width $=4^{\prime \prime}$.

under the $V$ magnitude 17.5 are listed in Table 3. Most of the stars with a strong color excess could be evolved but according to the absorption visible in Fig. 1 they should rather be in the background of the young region N26.

\section{Morphology and physical parameters}

Figure 4 shows monochromatic images through the [O III] $\lambda 5007, \mathrm{H} \beta, \mathrm{He}$ II $\lambda 4686$ and [O II] $\lambda 3727$ filters where the compact HII region N26 located to the south and the faint and circular nebula N25 to the north are well seen. The $\mathrm{H} \beta$ image of spatial resolution $1^{\prime \prime}$. 5 shows that N26 is composed of two nebular cores, A and B, separated only by $2^{\prime \prime}$ with a $\mathrm{A} / \mathrm{B}$ ratio of $\sim 5$. On the [O III] $\lambda 5007$ image the core A of $F W H M \sim 2$ '. $1(\sim 0.6 \mathrm{pc})$ is very compact and bright whereas $\mathrm{B}$ appears as a little bump. We notice $\sim 3^{\prime \prime}$ to the west of $\mathrm{A}$ an inclined faint low excitation filament showing up in the [O II] emission. On the $V$ image (Fig. 3), the brightest nebular core A is associated with the star $163 V_{\# 163}=15.41$, $B-V=0.31, U-B=-0.47$ and the fainter core B to the star $164 V_{\# 164}=16.42 B-V=0.42$. The HeII monochromatic image shows no trace of the $\lambda 4686$ emission noticed by Webster (1976). Contrary to the images, the spectra with a spatial resolution of $F W H M=2$.'1 and $33^{\prime \prime} 2$ along the $2^{\prime \prime}$ and $4^{\prime \prime}$ long-slit width do not allow us to separate the two cores of N26A-B. That is why in the physical parameter determination, such as spectral classification and computation of the abundances, only the sum of the two cores, $\mathrm{A}$ and $\mathrm{B}$, will be considered. The non-dereddened flux $F(\mathrm{H} \beta)$ through the $4^{\prime \prime}$ slit width is estimated to be $0.634 \times 10^{-12} \mathrm{erg} \mathrm{s}^{-1} \mathrm{~cm}^{-2}$, corresponding to a $\log F(\mathrm{H} \beta)=-12.20$ and a luminosity $\mathrm{L}_{\mathrm{H} \beta} \sim 0.35 \times 10^{36} \mathrm{erg} \mathrm{s}^{-1}$.

In Fig. 5, E-W profiles of N26A-B corresponding to the brightness distribution along the long-slit spectrum at $\lambda 4900$ and $\mathrm{H} \beta$ are shown, as well as those for the nebula N25. The cores A and B of N26A-B are not spatially resolved. In Table 4, Col. 2 presents the equatorial coordinates for J2000.0 with an accuracy of \pm 0 ". $3 \mathrm{~s}$ derived from the Denis astrometric calibration (Cioni et al. 2000), Col. 3 is the averaged $\mathrm{H} \alpha / \mathrm{H} \beta$ ratio of the whole nebula, Col. 4 the mean value of the $[\mathrm{O}$ III $] \lambda \lambda 5007+4959 / \mathrm{H} \beta$ ratio along the $2^{\prime \prime}$ long-slit, Col. 5 the $F W H M$ in arcsecs and parsecs $\left(1^{\prime \prime} \sim 0.30 \mathrm{pc}\right)$ of the objects in the $\mathrm{H} \beta$ line and Col. 6 the $V$ magnitude of the ionizing source obtained by 


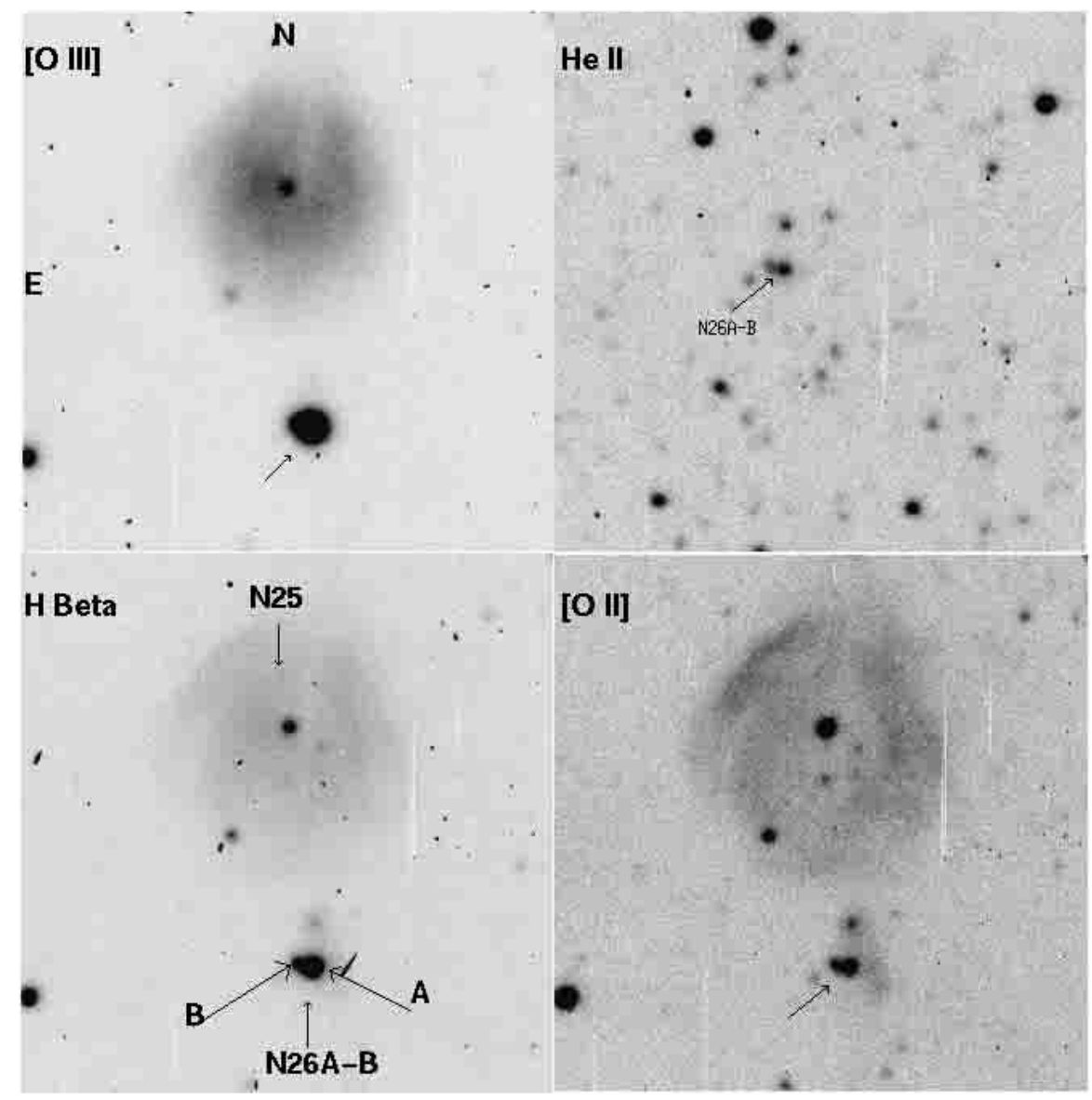

Fig. 4. Monochromatic images containing the nebula N25 (north) and the compact HII region N26A-B (south) in [O III] $\lambda 5007$, $\mathrm{H} \beta$, HeII $\lambda 4686$ and [O II] $\lambda 3727$ emission. On each frame N26A-B is indicated by an arrow, as well as the cores A and B in $\mathrm{H} \beta$. The size of each field corresponds to $86^{\prime \prime} \times 86^{\prime \prime}$.

imagery. For N26A-B, the magnitude derived is certainly underestimated due to the scattered light from dust responsible for the high extinction. The size of N26A-B is comparable to that of the trapezium young star cluster region of $\leq 1 \mathrm{pc}$ centered on a $\mathrm{O} 7 \mathrm{~V}$ star (Gagne et al. 1995).

\subsection{Extinction and $\mathrm{HI}$ emission}

The $\mathrm{H} \alpha / \mathrm{H} \beta$ ratio increases from west to east, reaching an $E(B-V)$ of 0.97 on the west wing of N26A-B (Fig. 6). The brightest core A has a mean extinction $E(B-V)=0.50$. If the absorption in $\mathrm{B}$ is due to dust there seems to be no correlation between the region of high excitation and high extinction, as in N88A (Testor \& Pakull 1985). The velocity found from the hydrogen lines $\sim 175 \pm 15 \mathrm{~km} \mathrm{~s}^{-1}$ seems to be correlated with the $167 \mathrm{~km} \mathrm{~s}^{-1} \pm 8 \mathrm{~km} \mathrm{~s}^{-1} \mathrm{HI}$ component of McGee et al. (1981). In the high-resolution HI survey (Stanimirovic et al. 1999) in the direction of N26A-B, the HI spectrum profile shows two peaks at $\sim 130$ and $\sim 160 \mathrm{~km} \mathrm{~s}^{-1}$. The column density corresponding to their sum is $1.04 \times 10^{22}$ atoms cm ${ }^{-2}, \sim 2-3$ times greater than the column density in the direction of N81 and N88A in the Shapley wing. The extinction in N26A-B contrasts with the one found in N25. From a E-W long-slit spec- trum of the whole nebula N25, centered on star \#237, we obtained a mean $E(B-V)=0.040$, very close to the SMC reddening value 0.034 given by McNamara \& Feltz (1980).

\subsection{Excitation and stellar content in N26A-B}

Through the $2^{\prime \prime}$ long-slit width, the integration of the whole nebula N26A-B gives for the [O III] $\lambda \lambda 4959+5007 / \mathrm{H} \beta$ intensity ratio a mean value of 6.5. Along the slit at the position of the core $\mathrm{A}$ this ratio can reach a maximum of $\sim 8$ and is lower in B (Fig. 6).

\subsubsection{Spectral type derived from $N_{L y c}$}

With reference to the paper by Devereux \& Scowen (1994), the Lyman continuum photon rate in photons per second, $N_{\text {Lyc }}$, produced by stars surrounded by an HII region can be derived from the flux of $\mathrm{H} \alpha$ photons $L_{\mathrm{H} \alpha}$ yielded by the ionization process in the nebula using the following equation:

$N_{\mathrm{Lyc}}=0.83 \times 10^{62} \times 10^{E(B-V)} F_{\mathrm{H} \alpha} D^{2}$

which also takes into account the extinction. $\mathrm{D}$ is the distance in Mpc. The $N_{\text {Lyc }}$ value thus obtained can then be expressed in terms of equivalent number of ionizing 


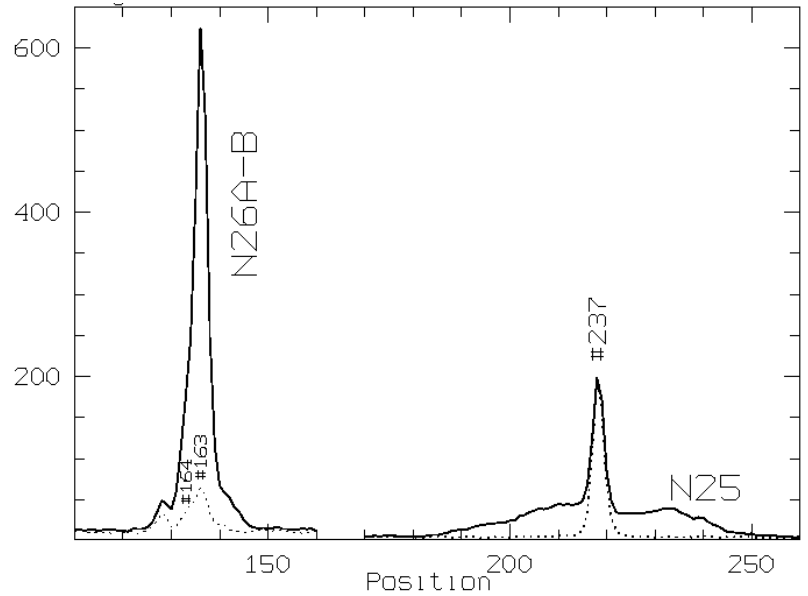

Fig. 5. E-W relative brightness distribution obtained through a $2^{\prime \prime}$ long-slit at $\lambda 4880$ (dotted line) and $\mathrm{H} \beta$ (thick line) of the region N26A-B and the neigbouring extended HII region N25.

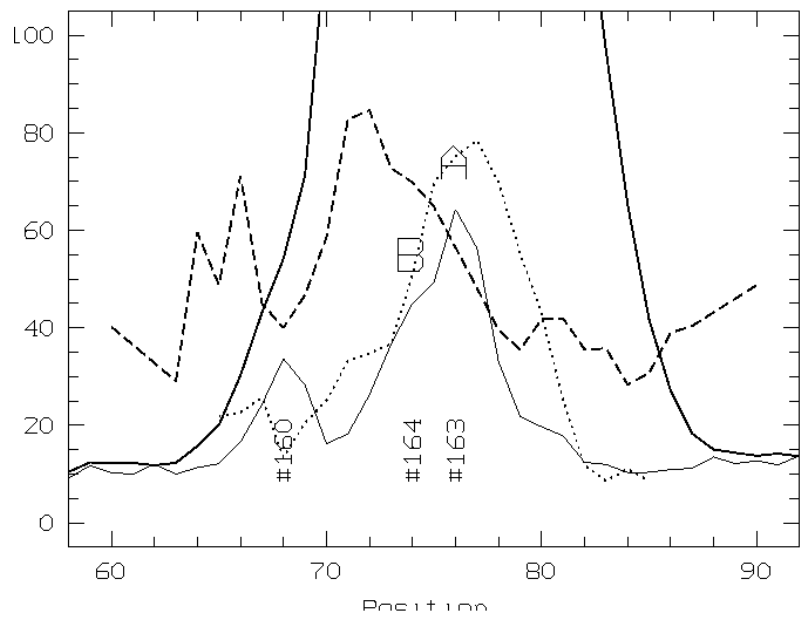

Fig. 6. Along the E-W $2^{\prime \prime}$ long-slit, the thin line corresponds to the continuum brightness near $\mathrm{H} \beta$ of the two unresolved stars \#163 and \#164 in the A and B cores and the thick line shows the wings of the $\mathrm{H}_{\alpha}$ brightness profile. The dashed line corresponds to the $\mathrm{H} \alpha / \mathrm{H} \beta$ and the dotted line to the [O III] $\lambda \lambda 5007+4959 / \mathrm{H} \beta$ ratios multiplied in the $Y$ scale by a factor 10 .

stars of a given spectral type. Using for the entire nebula N26A-B a mean $E(B-V)$ of 0.40 and a $F(\mathrm{H} \beta)$ of $\sim 0.634 \times 10^{-12} \mathrm{erg} \mathrm{s}^{-1} \mathrm{~cm}^{-2}$, the $N_{\mathrm{Lyc}}$ derived is $2.78 \times 10^{48} \nu \mathrm{s}^{-1}$ indicating that the whole nebula should be excited by a source equivalent to an $08.5 \mathrm{~V}$ star (Panagia 1973) or O9 V (Vacca et al. 1996). With an A/B ratio of $\sim 5$ the core $\mathrm{A}$ should be excited by a star of type O9-09.5 V and the core B by a type B0-B0.5 V.

\subsubsection{Spectral type derived from spectroscopy}

We also determined the spectral type of the ionizing source by spectroscopy, comparing our data with Walborn \& Fitzpatrick's (1990) atlas of digital spectra. The neighbouring sky is subtracted but not the strong nebular contribution. With a spatial resolution $\geq 2$ ". 5 , the subtraction

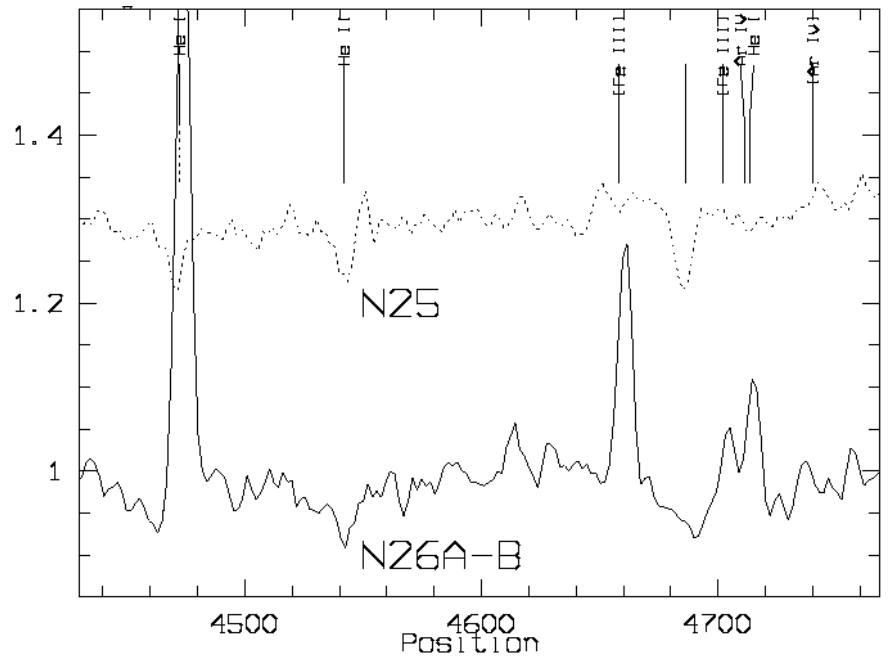

Fig. 7. E-W rectified spectra in a range $4450-4750 \AA$ of the central ionizing source in N26A-B and in N25.

of the nebula at both sides of the star is not possible and does not allow the discrimination between the observed nebular and stellar profiles of HeI $\lambda 4471$.

Despite a bad $S / N$ continuum, the $\lambda 4200, \lambda 4541$ and $\lambda 4686$ absorption lines are detectable. Figure 7 shows in a range $4450-4750 \AA$ the integrated spectrum of N26A-B composed of the spatially unresolved stars \#163 and \#164 of one magnitude fainter. The contribution of the $\lambda 4471$ line is not visible and the integrated spectrum seems to correspond to a type O7-09 V star whose ionizing flux is slightly in excess of that derived from the $N_{\text {Lyc }}$ flux.

The central star of the extended nebula N25 to the north (Fig. 7) is reclassified as an $\mathrm{O} 8 \mathrm{~V}$ star rather than O9 V (Hutching \& Thomson 1988).

\subsection{Temperature and density}

Physical parameters were computed using the 1996 version of the ABELION program developed by Stasińska. This program uses a five-level atom and the atomic data are described in Stasińska \& Leitherer (1996). The emission-line intensities were corrected for reddening using the observed $\mathrm{H} \alpha / \mathrm{H} \beta$ ratio and the Seaton (1979) extinction law. Four electron temperatures were derived, associated with different ionization regions in the nebula. These temperatures were computed from the following line intensity ratios: $T_{\mathrm{e}}[\mathrm{O}$ III $] \lambda 4363 / \lambda \lambda(4959+5007)$, $T_{\mathrm{e}}[\mathrm{N} \mathrm{II}] \lambda 5755 / \lambda \lambda(6548+6584), T_{\mathrm{e}}[\mathrm{O}$ II $] \lambda 3727 / \lambda \lambda(7319+$ $7330)$ and $[\mathrm{S}$ III $] \lambda 6312 / \lambda 9532$. Two electron densities were determined from the $[\mathrm{S}$ II $] \lambda 6717 / \lambda 6731$ and [Cl III $] \lambda 5517 / \lambda 5537$ ratios.

In the determination of the physical parameters due to the $S / N$ lines, only the [O III] $\lambda 4363 / \lambda \lambda(4959+5007)$ and $[\mathrm{S} \mathrm{II}] \lambda 6717 / \lambda 6731$ ratios were used.

The mean electron densities, $N_{\mathrm{e}}$, and the mean electron gas temperatures $T_{\mathrm{e}}[\mathrm{O}$ III], are shown in Table 5 as well as temperatures and densities obtained with the other ratios as terms of comparison. 
Table 5. Forbidden and permitted emission line intensities in N26A-B and N25.

\begin{tabular}{|c|c|c|c|c|c|c|c|}
\hline & Ion & $\begin{array}{l}I_{\lambda} / \mathrm{H}_{\beta} \\
\mathrm{N} 26 \mathrm{~A}-\mathrm{B}^{n}\end{array}$ & $S / N$ & $\begin{array}{l}I_{\lambda} / \mathrm{H}_{\beta} \\
\text { N26A-B }\end{array}$ & $S / N$ & $\begin{array}{l}I_{\lambda} / \mathrm{H}_{\beta} \\
\mathrm{N} 25^{n}\end{array}$ & $S / N$ \\
\hline 3727 & {$[\mathrm{O} \quad \mathrm{II}]$} & 0.4355 & 104 & 1.0682 & 156 & 1.6740 & 30 \\
\hline 3868 & [Ne III] & 0.1878 & 56 & 0.2311 & 53 & 0.2088 & 12 \\
\hline 4072 & {$[\mathrm{~S}$ II $]$} & 0.0059 & 3 & 0.0070 & 4 & & \\
\hline 4076 & {$[\mathrm{~S}$ II] } & 0.0025 & 1 & & 3 & & \\
\hline 4100 & $\mathrm{H} \delta$ & 0.2090 & 66 & 0.2270 & 60 & 0.2580 & 18 \\
\hline 4287 & {$[\mathrm{Fe} \mathrm{II}]$} & 0.0025 & 4 & & & & \\
\hline 4340 & $\mathrm{H} \gamma$ & 0.3793 & 129 & 0.4023 & 137 & 0.475 & 25 \\
\hline 4363 & {$[\mathrm{O}$ III $]$} & 0.0441 & 26 & 0.0426 & 19 & 0.0310 & 7 \\
\hline 4471 & $\mathrm{He} \mathrm{I}$ & 0.0308 & 16 & 0.0303 & 13 & 0.0312 & 6 \\
\hline 4658 & {$[\mathrm{Fe} \mathrm{III}]$} & 0.0127 & 9 & 0.0221 & 9 & & \\
\hline 4701 & {$[\mathrm{Fe} \mathrm{III}]$} & 0.0036 & 2 & & & & \\
\hline 4711 & {$[\mathrm{Ar}$ IV $]+\mathrm{He} \mathrm{I}$} & 0.0068 & 3 & 0.0075 & 6 & 0.0327 & 4 \\
\hline 4740 & [Ar IV] & & & & & 0.0046 & 1 \\
\hline 4754 & [Fe III] & 0.0019 & 3 & 0.0012 & 2 & & \\
\hline 4769 & {$[\mathrm{Fe} \mathrm{III}]$} & 0.0022 & 3 & & & & \\
\hline 4861 & $\mathrm{H} \beta$ & 1. & 331 & 1. & 415 & 1. & 38 \\
\hline 5007 & [O III $]$ & 4.9000 & 1000 & 4.2367 & 1184 & 3.1900 & 65 \\
\hline 5056 & Si II & 0.0015 & 2 & & & & \\
\hline 5200 & {$[\mathrm{NI}]$} & 0.0090 & 10 & 0.0126 & 5 & 0.0143 & 3 \\
\hline 5270 & {$[\mathrm{Fe} \mathrm{III}]$} & 0.0082 & 8 & 0.0088 & 9 & & \\
\hline 5299 & O I & 0.0007 & 6 & & & & \\
\hline 5518 & [Cl III] & 0.0046 & 5 & & & & \\
\hline 5538 & {$\left[\begin{array}{ll}\mathrm{Cl} & \mathrm{III}\end{array}\right]$} & 0.0042 & 4 & & & & \\
\hline 5755 & {$[\mathrm{~N} \mathrm{II}]$} & 0.0026 & 3 & 0.0028 & 4 & 0.0060 & 2 \\
\hline 5876 & $\mathrm{He} \mathrm{I}$ & 0.1784 & 134 & 0.1589 & 133 & 0.1131 & 20 \\
\hline 5958 & Si II & 0.0025 & 4 & 0.0056 & 3 & & \\
\hline 5979 & Si II & 0.0019 & 3 & 0.0047 & 3 & 0.0141 & \\
\hline 6046 & O I & 0.0025 & 3 & 0.0028 & 2 & & \\
\hline 6300 & {$[\mathrm{O}$ I] } & 0.0166 & 27 & 0.0154 & 23 & 0.0450 & 5 \\
\hline 6311 & [S III] & 0.0335 & 35 & 0.0272 & 36 & 0.0211 & 4 \\
\hline 6347 & Si II & 0.0036 & 3 & 0.0034 & 3 & 0.0086 & 3 \\
\hline 6364 & {$[\mathrm{O}$ I $]$} & 0.0062 & 7 & 0.0082 & 6 & 0.0078 & 2 \\
\hline 6563 & $\mathrm{H} \alpha$ & 4.8010 & 1300 & 4.2917 & 1486 & 2.8600 & 62 \\
\hline 6584 & {$[\mathrm{NII}]$} & 0.1081 & 115 & 0.1440 & 173 & 0.0953 & 13 \\
\hline 6678 & He I & 0.0536 & 70 & 0.0489 & 58 & 0.0340 & 9 \\
\hline 6717 & {$[\mathrm{~S}$ II $]$} & 0.0518 & 80 & 0.0788 & 93 & 0.1283 & 14 \\
\hline 6731 & {$\left[\begin{array}{ll}\mathrm{S} & \mathrm{II}\end{array}\right]$} & 0.0707 & 91 & 0.0925 & 104 & 0.0883 & 12 \\
\hline 7002 & $\mathrm{O} \mathrm{I}+\mathrm{Ar} \mathrm{V}$ & 0.0024 & 4 & 0.0026 & 3 & & \\
\hline 7135 & [Ar III] & 0.1686 & 176 & 0.1143 & 144 & 0.0913 & 11 \\
\hline 7256 & O I & 0.0049 & 4 & 0.0042 & 4 & & \\
\hline 7325 & {$[\mathrm{O} \quad \mathrm{II}]^{a}$} & 0.2314 & 160 & 0.1667 & 163 & 0.0850 & 9 \\
\hline 9069 & [S III] & & & 0.36 & 40 & & \\
\hline 9532 & {$[\mathrm{~S}$ III] } & & & 1.03 & 50 & & \\
\hline $\log F(\mathrm{H} \beta)$ & $\mathrm{ergcm}^{-2} s^{-1}$ & -12.30 & & -12.20 & & -12.33 & \\
\hline$c(\mathrm{H} \beta)$ & & $0.70 \pm 0.02$ & & $0.56 \pm 0.02$ & & $0.020 \pm 0.05$ & \\
\hline$E W(\mathrm{H} \alpha)$ & & 900 & & 1100 & & & \\
\hline$N_{\mathrm{e}}[\mathrm{S} \mathrm{II}]$ & $\left(\mathrm{cm}^{-3}\right)$ & $1850 \pm 50$ & & $1130 \pm 30$ & & $100 \pm 20$ & \\
\hline$N_{\mathrm{e}}[\mathrm{Cl}$ III $]$ & $\left(\mathrm{cm}^{-3}\right)$ & $1750 \pm 500$ & & & & & \\
\hline $\begin{array}{l}T_{\mathrm{e}}(\mathrm{K}) \\
T_{\mathrm{e}}(\mathrm{K})\end{array}$ & {$\left[\begin{array}{ll}0 & 11\end{array}\right]$} & $12200 \pm 200$ & & $\begin{array}{l}15400 \pm 400 \\
12500 \pm 200\end{array}$ & & $11500 \pm 400$ & \\
\hline$T_{\mathrm{e}}(\mathrm{K})$ & {$\left[\begin{array}{ll}\mathrm{N} & \mathrm{II}\end{array}\right]$} & $13900 \pm 2500$ & & $12500 \pm 1500$ & & $11000+400$ & \\
\hline$T_{\mathrm{e}}(\mathrm{K})$ & [S III] $]$ & & & $13900 \pm 1000$ & & & \\
\hline
\end{tabular}

${ }^{n}$ Period 1991: slit width $=2^{\prime \prime},{ }^{l}$ Period 1997 and 90: slit width $=4^{\prime \prime}(3700-7400 \AA)$ and $3^{\prime \prime}$ for NIR. ${ }^{a}$ sum: [OII] $\lambda 7319+[\mathrm{OII}] \lambda 7330$.

\subsection{Mass of ionized gas}

Density diagnostics, such as the [SII] line ratio, measure the density of the matter $N_{\mathrm{e}}$ ([S II $\left.]\right)$ while the mean elec- tron density $<N_{\mathrm{e}}>$ (Pereira 2000) that can be derived from $N_{\mathrm{Lyc}}$ takes into account the empty medium as well. 
Table 6. Element abundances in N26A-B and N25 in the form $(12+\log [N(\mathrm{X}) / N(\mathrm{H})])$.

\begin{tabular}{llllllll}
\hline \hline Name & $\mathrm{O}$ & $\mathrm{N}$ & $\mathrm{Ne}$ & $\mathrm{Ar}$ & $\mathrm{S}$ & $\mathrm{He}$ & $\mathrm{C}(\mathrm{H} \beta)$ \\
\hline $\mathrm{N} 25$ & $8.00 \pm 0.05$ & $6.64 \pm 0.02$ & $7.23 \pm 0.04$ & $5.96 \pm 0.05$ & & $10.93 \pm 0.02$ & 0.02 \\
$\mathrm{~N} 26 \mathrm{~A}-\mathrm{B}^{97}$ & $7.98 \pm 0.03$ & $6.59 \pm 0.03$ & $7.26 \pm 0.03$ & $5.76 \pm 0.04$ & $6.31 \pm 0.03$ & $10.92 \pm 0.02$ & 0.56 \\
$\mathrm{~N}^{9} \mathrm{~A}-\mathrm{B}^{91}$ & $7.99 \pm 0.02$ & $6.69 \pm 0.03$ & $7.18 \pm 0.03$ & $5.89 \pm 0.03$ & & $10.92 \pm 0.01$ & 0.72 \\
$\mathrm{SMC}^{R e}$ & 7.96 & 6.55 & 7.17 & 5.72 & 6.32 & 10.91 & \\
$\mathrm{SMC}^{G}$ & 8.0 & 6.5 & 7.2 & 5.9 & 6.3 & & \\
$\mathrm{~N}^{K} 8 \mathrm{~A}^{K}$ & $8.05 \pm 0.08$ & $6.59 \pm 0.08$ & $7.26 \pm 0.05$ & $5.69 \pm 0.07$ & $6.42 \pm 0.11$ & 10.91 & \\
\hline
\end{tabular}

${ }^{G}$ Garnett (1999), ${ }^{R e}$ Reyes (1999), ${ }^{K}$ Kurt et al. (1999).

${ }^{91}$ Abundances: computed from the average of 3 frames obtained in 1991.

${ }^{97}$ Abundances: computed from an optical single frame obtained in 1997 and a NIR in 1990.

where $\alpha_{\mathrm{B}}$ is the recombination coefficient for Case $\mathrm{B}$ (Osterbrock 1989) and $D=1.2 \mathrm{pc}$ the adopted diameter of the HII region (slit width $\left.=4^{\prime \prime}\right)$.

Assuming that the gas is distributed in clumps of uniform density, the ratio of these two measures of the density gives the filling factor $f$

$f=\frac{<N_{\mathrm{e}}>}{N_{\mathrm{e}}([\mathrm{SII}])}$.

The mass of ionized gas $\left(M_{\mathrm{HII}}\right)$, in units of solar mass, is derived from $N_{\text {Lyc }}$ using the relation

$M_{\mathrm{HII}}=\frac{4 \pi r_{\mathrm{obs}}^{3}<N_{\mathrm{e}}>m_{\mathrm{H}}}{3}$

where $\mathrm{m}_{\mathrm{H}}$ is the mass of the hydrogen atom and $r_{\text {obs }}$ the observed radius. We find a filling factor $f=0.6$ and a mass of ionized gaz of $\sim 13 M_{\odot}$.

\subsection{Nature of $N 26 A-B$}

In Fig. 8a of Testor \& Pakull (1985) N26A-B lies at the frontier between planetary nebulae and HII regions. However, the mass of $\sim 13 M_{\odot}$ should exclude the possibility that N26A-B is a planetary nebula.

\section{Chemical abundances and summary of results}

Like the other "compact HII blobs", N26A-B is well adapted to computations of abundances. It is bright and corresponds in size to a Stromgren sphere entering partially or completely in the $2^{\prime \prime}$ and 4 " slit of the spectrograph.

\subsection{Ionic and elemental abundances}

Using the derived $T_{\mathrm{e}}[\mathrm{O} \mathrm{III}]$ and $N_{\mathrm{e}}[\mathrm{S}$ II $]$ values for the nebula, the ionic and total elemental abundances have been calculated and the results are presented in Table 6 . As a term of comparison the table also contains the latest average HII abundances in the SMC derived by Reyes (1999) and Garnett (1999), the average abundances in N88A derived from the intensive study of Kurt et al. (1999) and the abundances in the extended HII region N25 computed in this work. Small discrepancies are noticed between the abundances derived from 1991 and 1997 observations as well as between the mean abundances given by Reyes (1999) and Garnett (1999). Despite our small error bars it is difficult to consider such a scatter as a physical reality. However, compared to the average HII abundances of the SMC, N26A-B seems to present a slight overabundance of $\mathrm{N}$. This excess is also visible in N25.

\subsection{Results}

SMC N26A-B is a small compact HII region located at the border of an HI cloud south of the bar. On the monochromatic images the region is composed of a bright core $\mathrm{A}$ of $F W H M \sim 0.6 \mathrm{pc}$ and a fainter one, B, separated by $\sim 0.6$ pc.

Low-resolution long-slit spectra with the ESO $1.5 \mathrm{~m}$ telescope show:

- a radial velocity of $175 \pm 15 \mathrm{~km} \mathrm{~s}^{-1}$, similar to the $170 \mathrm{~km} \mathrm{~s}^{-1}$ of the HI cloud component;

- evidence for substantial dust, mainly in and east of B provided by the $\mathrm{H} \alpha / \mathrm{H} \beta$ ratio reaching a maximum value of 8.5 ;

- a high excitation level for HII regions: [O III] $\lambda \lambda 5007+4959 / \mathrm{H} \beta$ up to 8 in $\mathrm{A}$.

- that the main source responsible for the high excitation of the nebula is centered on $\mathrm{A}$ and has an equivalent spectral type in a range $\mathrm{O} 7-\mathrm{O} 9 \mathrm{~V}$.

- an [O III] electron temperature of $\sim 12500 \mathrm{~K}$, and an electron density of $\sim 1130 \mathrm{~cm}^{-3}$ for the whole nebula, reaching $\sim 1850 \mathrm{~cm}^{-3}$ in A through a slit aperture of $2^{\prime \prime} \times$ $2^{\prime \prime}$ and a mass of the total ionizing gas estimated at $13 M_{\odot}$.

The analysis of N26A-B indicates that the gaseous phase abundance of $\mathrm{He}, \mathrm{O}, \mathrm{N}, \mathrm{Ne}, \mathrm{S}$ and $\mathrm{Ar}$ presents some discrepancies compared to the two latest SMC average abundances themselves displaying differences. It is not possible to give them a significative physical reality, except for $\mathrm{N}$, which seems overabundant.

The morphological and spectral characteristics of N26A-B place it in the category of compact HII regions that we call blobs in the Magellanic Clouds and among them, N26A-B is the faintest and the smallest. 
Our results point out that more observations in spectrophotometry and imagery with large ground-based telescopes and the HST are still needed to increase the $S / N$ and the spatial resolution of the nebula and its faint embedded exciting sources. These observations should especially establish if the main sources responsible for the ionization are single massive stars or components of tight clusters, refine the spectral type classification and resolve the abundance discrepancies. The new region N26A-B increases the sample of compact HII regions of star formation $(F W H M \leq 2 \mathrm{pc})$ in an environment of low metallicity. A serious comparison with other similar regions of the same or different metallicity, such as Orion, the most well-studied HII region (type III-IV) in our galaxy, appears possible and should improve the understanding of such star formation sites.

\section{References}

Azzoppardi, M., \& Vigneau, J. 1979, A\&AS, 35, 353

Caplan, J., \& Deharveng, L. 1986, A\&A, 155, 297

Cioni, M.-R., et al. 2000, A\&AS, 144, 235

Davies, R. D., Elliot, K. H., \& Meaburn, F. 1976, MNRAS, 81, 89

Devereux, N. A., \& Scowen, P. A. 1994, AJ, 108, 1244

Gagne, M., Caillaut, J. P., \& Stauffer, J. R. 1995, ApJ, 445, 280

Garnett, D. 1999, IAU Symp, 190, 266

Graham, J. A. 1982, PASP, 94, 244

Henize, K. G. 1956, ApJS, 2, 315

Heydari-Malayeri, M., \& Testor, G. 1982, A\&A, 111, L11

Heydari-Malayeri, M., \& Testor, G. 1983, A\&A, 118, 116

Heydari-Malayeri, M., \& Testor, G. 1985, A\&A, 144, 98

Heydari-Malayeri, M., \& Testor, G. 1986, A\&A, 162, 180
Heydari-Malayeri, M., Le Bertre, T., \& Magain, O. 1988, A\&A, 195,230

Heydari-Malayeri, M., Van Drom, E., \& Leisy, P. 1990, A\&A, 240,481

Hummer, D. G., \& Storey, P. J. S. 1987, MNRAS, 224, 801

Hutchings, J. B., \& Thomson, I. B. 1988, ApJ, 331, 294

Israel, F. P., Johansson, L. E. B., Lequeux, J., et al. 1993, A\&A, 276, 25

Kurt, C., Dufour, R., Garnett, D., et al. 1999, ApJ, 518, 246, 261

McGee, R. X., \& Newton, L. M. 1981, Proc. Astron. Soc. Australia, 4, 189

McNamara, D. H., \& Feltz, K. A. 1980, PASP, 92, 587

Newberry, M. V. 1994, CCDA, 1, 34

Osterbrock, D. E. 1989, Astrophysics of Gaseous Nebulae and active Galactic Nuclei (San Francisco: Freeman)

Oke, J. B. 1974, ApJS, 27, 21

Pagel, B. E. J., Edmunds, M. G., Fosbury, R. A. E., \& Webster, B. L. 1978, MNRAS, 184, 569

Panagia, N. 1973, AJ, 78, 929

Pereira, C. B. 2000, AJ, 119, 63

Reyes, C. 1999, IAU Symp, 190, 282

Stasińska, G., \& Leitherer, C. 1996, ApJS, 107, 661, 66, 257

Stetson, P. B. 1987, PASP, 99, 191

Seaton, M. J. 1979, MNRAS, 187, 73

Skillman, E. D. 1985, ApJ, 290, 449

Stanimirovic, S., Stavley-Smith, L., Dickey, J. M., Sault, R. J., \& Snowden, S. L. 1999, MNRAS, 302, 417

Stanimirovic, S., Stavley-Smith, L., van der Hulst, J. M., et al. 2000, MNRAS, 315, 791

Testor, G., \& Pakull, M. 1985, A\&A, 145, 170

Vacca, W. D., Garmany, C. D., \& Shull, J. M. 1996, ApJ, 460, 914

Walborn, N., \& Fitzpatrick, E. 1990, PASP, 102, 379

Webster, B. L. 1976, MNRAS, 174, 513

Wilcots, E. M. 1994, AJ, 108(4), 1674 